\title{
Dégradation des sols et stratégies coopératives en Méditerranée : la pression sur les ressources naturelles et les stratégies de développement durable
}

Rabah Lahmar ${ }^{1}$
Alain Ruellan

${ }^{1}$ Centre de coopération internationale en recherche agronomique pour le développement (Cirad), Unité mixte de recherche (UMR) "SYSTEM SupAgro",

Bâtiment 27,

2, place Viala,

34060 Montpellier

<rabah.lahmar@cirad.fr>

2 2, Bd Berthelot,

34000 Montpellier

<ruellan@agropolis.fr>

\begin{abstract}
Résumé
L'érosion et la salinisation sont apparues très tôt en Méditerranée, dès l'introduction de l'agriculture et de l'irrigation. Aujourd'hui, dans tous les pays méditerranéens d'autres formes de dégradations sont reconnues: elles sont encore peu documentées et leurs sévérités et risques ne sont pas évalués. L'agriculture n'est plus la seule cause des dégradations des sols : l'urbanisme, l'industrie, les transports, l'artisanat et les loisirs y contribuent. L'état des lieux suggère que les dégradations des sols sont très rapides en Méditerranée; dans certaines situations, les seuils de l'irréversibilité sont déjà atteints, voire dépassés. La complexité des phénomènes, leurs interrelations et imbrications, leurs impacts parfois transfrontaliers, exigent des efforts de recherche dépassent le cadre et les moyens des États. Une politique méditerranéenne en faveur des sols est donc nécessaire.
\end{abstract}

Mots clés : coopération ; dégradation du sol ; impact sur l'environnement ; région méditerranéenne.

Thèmes : sols ; ressources naturelles et environnement ; territoire, foncier, politique agricole et alimentaire.

\begin{abstract}
Soil degradation in the Mediterranean region and cooperative strategies

Soil erosion and salinisation emerged very early in the Mediterranean region along with the introduction of agriculture and irrigation. Nowadays, in all Mediterranean countries, other forms of soil degradation are obvious: they are still poorly documented and their severity and risks have not been assessed. Agriculture is no longer the only cause of soil degradation: urbanism, industry, transportation, handicraft and leisure also contribute. The state of the art inventory suggests that soil degradation is very swift in the Mediterranean region and that in some situations the thresholds of irreversibility have already been reached or even exceeded. The complexity of the phenomena, with their inter-relations and overlapping and their sometimes trans-border impacts, call for research efforts exceeding the framework and means of the separate States. A Mediterranean policy in favour of soils is therefore necessary.
\end{abstract}

Key words: Mediterranean region; soil degradation; cooperation; environmental impact.

Subjects: soil, natural resources and environment; territory land use, agricultural and food production policy.

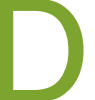
ans le passé, la fertilité des sols méditerranéens a suscité des convoitises : l'antique Rome avait bien fait de l'Afrique du Nord un grenier à blé ! De nos jours leur dégradation pose problème et elle pourrait compromettre l'avenir de bien des pays. 
La dégradation des sols est une problématique globale (Oldeman et al., 1991 ; Scherr, 1999 ; Eswaran et al., 2001 ; Eswaran et Reich, 2002) et complexe. La complexité tient à : i) la diversité des formes de dégradations, leurs interrelations et imbrications, leur extension, sévérité et réversibilité (Van Lynden, 1995 ; Scherr 1999); ii) la diversité des facteurs de dégradation, qui peuvent être naturels mais surtout anthropiques (Oldeman et al., 1991 ; Van Lynden 1995) ; iii) la difficulté d'établir des indicateurs, des seuils et des relations de cause à effet entre une forme de dégradation et ses impacts (Eswaran et al., 2001 ; Scherr 1999) ; et iv) la difficulté d'évaluer les impacts sociaux, économiques et environnementaux immédiats et sur le long terme, à l'endroit où les dégradations se produisent et au-delà (Van Lynden 1995; Malik, 1998 ; Scherr, 1999 ; Eswaran et al., 2001 ; Lipper et Osgood, 2001). Par ailleurs, les différentes perceptions disciplinaires et utilitaires du rôle du sol ont conduit à plusieurs définitions de la dégradation des sols (Van Lynden, 1995; Malik, 1998 ; Eswaran et al., 2001). Tout cela a contribué à retarder la prise de conscience de la gravité du problème et de la nécessité de sa prise en charge.

Après un rapide examen des différents types de dégradation des sols en Méditerranée, de leurs origines et développements, nous présenterons, sur une base bibliographique, l'état des connaissances actuelles relatives à chacune de ces dégradations, et nous en tirerons les conséquences en termes d'arguments pour une stratégie méditerranéenne en faveur des sols.

\section{Les différents types de dégradation des sols \\ en Méditerranée}

La dégradation des sols des pays méditerranéens est un problème ancien, et d'autant plus sérieux que la pression démographique qui ne cesse d'augmenter s'exerce sur un milieu naturel fragile. Dans toutes les évaluations mondiales de la dégradation des sols (Eswaran et al., 2001 ; Eswaran et Reich, 2002 ; http:// www.fao.org/landandwater/agll/glasod/ glasodmaps.jsp) le Bassin méditerranéen se singularise. En effet, les régions méditerranéennes cumulent les facteurs propices à la dégradation des sols : un couvert végétal souvent clairsemé, l'agressivité des pluies et des vents, des roches souvent d'érosion facile, une topographie ondulée facilitant l'érosion des sols en pente, des sols souvent peu épais, l'aridité favorisant l'accumulation des sels solubles. À cela s'ajoute une occupation humaine des plus anciennes avec une pratique continue de l'agriculture et de l'élevage depuis le néolithique. Les sols méditerranéens feraient ainsi partie des sols les plus anthropisés de la planète (Boulaine, 1961 ; Yaalon, 1997).

Les problèmes d'érosion et de salinisation sont apparus très tôt en Méditerranée (Griesbach, 1993; Yaalon, 1997). L'érosion hydrique a accompagné la déforestation entamée dès l'introduction de l'agriculture. Elle s'est fortement accélérée aux époques grecque et romaine, puis s'est poursuivie à des ampleurs variables aux époques ultérieures. La forêt originelle couvrait $40 \%$ de l'espace; elle n'en couvre que $4 \%$ actuellement (Yaalon, 1997). La salinisation est apparue avec l'irrigation. L'érosion et la salinisation auraient contribué, si ce n'est causé, le déclin de grandes civilisations en Mésopotamie, Egypte, Afrique du Nord, Grèce et Italie (Griesbach, 1993).

D'autres formes de dégradation du sol sont reconnues : diminution de la matière organique du sol et de son activité biologique ; destruction de la structure du sol, tassement du sol et formation et croûtes superficielles ; baisse de la fertilité chimique du sol ; pollutions ; acidité ; diminution des surfaces de terres arables du fait de l'urbanisme et des infrastructures.

Toutes ces dégradations sont induites par l'activité humaine. Elles existent dans tous les pays méditerranéens. Leur extension géographique, leur ampleur et leurs impacts restent cependant du domaine d'appréciation des experts. L'érosion et la salinisation ont fait l'objet de beaucoup de recherches alors que les autres formes de dégradation n'ont jusqu'à présent bénéficié que de peu d'études scientifiques, très dispersées.

Dans nombre de pays méditerranéens, les études et reconnaissances pédologiques ont débuté bien avant 1950. Vers 1965, la majorité des pays disposaient de cartes de reconnaissance des sols à des échelles allant du 1:200000 au 1:1 000 000. À la fin des années 1980, tous les pays méditerranéens avaient des cartes de synthèse allant du 1:500 000 au
1:2000 000 (Griesbach, 1993). Cependant, pour des raisons de conception et d'échelle et parce que la pédodiversité est forte en Méditerranée (Costantini et al., 2002), tous ces documents se sont avérés peu exploitables au regard des problèmes de dégradation du sol qui nécessitent plus de détails et des données quantitatives réactualisées périodiquement. Ces considérations, entre autres, ont amené certains pays méditerranéens à réactualiser et compléter leurs cartes et à en informatiser l'usage (Darwish et al., 2002 ; Nachtergaele et Oldeman, 2002 ; Zdruli et al., 2002).

\section{Aperçu \\ sur les différents types de dégradations des sols en Méditerranée}

\section{Salinisation}

Aujourd'hui, toutes les zones irriguées de Méditerranée sont concernées par les problèmes de salinisation des sols (Al Qudah, 2001 ; Badraoui et Stitou, 2001 ; Darwish 2001; Dinç et al., 2001; Dudeen, 2001; Hadjiparaskevas, 2001 ; Hamdi et Abdelhafez, 2001 ; Ilaiwi, 2001 ; Mtimet, 2001; Ramdane, 2001; Vela, 2001 ; Zdruli et Lushaj, 2001 ; Vacca et al., 2002). Le phénomène dépasse le cadre classique des périmètres irrigués continentaux pour atteindre les étroites bandes côtières comme en Albanie (Zdruli et Lushaj, 2001) et dans les îles (Hadjiparaskevas, 2001; Vela, 2001 ; Vacca et al., 2002). Au Liban, la salinité apparaît même sous les serres (Darwish, 2001). L'extension d'une irrigation mal maîtrisée à des terres plus ou moins " aptes " à l'irrigation, l'utilisation d'eaux de moindre qualité, y compris les eaux d'intrusions marines, et l'inexistence ou l'inefficience du drainage concourent à étendre la salinité des sols, parfois de manière très rapide. Cependant, Dosso (1980) et Ilaiwi (2001) montrent que malgré l'évidence historique de l'irrigation en Syrie entre 4000 et 3000 avant J.-C, la salinisation n'est apparue de manière notable que dans les années 1950, avec le développement de l'irrigation du coton sans drainage. Au Maghreb, dans des périmètres irrigués par pivot, 
Daoud et Halitim (1994) et Badroui et al. (1998) ont montré qu'en moins de 10 ans d'irrigation la salinité pouvait atteindre des niveaux contraignants. Par ailleurs, Mhiri et al. (1998) notent que la rétention croissante des eaux de surface sur le continent et leur utilisation et réutilisation en agriculture s'accompagne d'une concentration de plus en plus forte de sels dans le système hydropédologique ; ils qualifient le processus d'endoréisation anthropique.

Le manque d'une cartographie précise des sols salés et d'un suivi de l'évolution temporelle de la salinité dans les zones irriguées ne permet pas de juger des risques ni d'ailleurs des efforts entrepris de restauration des sols salés, et encore moins d'anticiper le phénomène avec l'extension "forcée " de l'irrigation.

\section{Érosion hydrique}

L'érosion hydrique est largement répandue en Méditerranée et continue de s'amplifier particulièrement en zones de pentes (Boukheir et al., 2001). En Albanie (Zdruli et Lushaj, 2001), au Maroc (Badraoui et Stitou, 2001) et en Sardaigne (Vacca et al., 2002), elle semble avoir atteint des niveaux critiques. En plus de la déforestation, du surpâturage et du labour, habituellement cités comme causes, Vacca et al. (2002) ajoutent pour ce qui concerne la Sardaigne : i) la pratique du feu pour l'extension des pâturages ; ii) l'accroissement démesuré du cheptel ovin et avec lui la compaction du sol ; iii) la plantation d'espèces exotiques. Des mesures à la parcelle ont révélé des pertes de sol sous Eucalyptus deux fois plus importantes que dans les pâturages abandonnés et six fois plus que dans les maquis incendiés. Pardini et al. (2003) comparent en Catalogne les pertes dans des parcelles laissées à la recolonisation naturelle $(5,25$ et 50 ans) et un reboisement de pins de 50 ans d'âge et des plantations de vigne et d'olivier insuffisamment gérées. Ces auteurs concluent que la reforestation non suivie d'une gestion suffisante peut affecter négativement les propriétés du sol et sa réponse à l'action érosive du ruissellement.

Roose et De Noni (1998) ont analysé l'apport de la recherche à la lutte antiérosive, y compris l'expérience méditerranéenne des années 1940 à 1980 de défense et restauration des sols. Ces auteurs concluent à un bilan mitigé et au besoin d'une nouvelle approche. Dans le même sens, Lavee et al. (2004), tirant les leçons de 20 ans de mesure de l'érosion en Méditerranée, notent que les connaissances acquises ne permettent pas d'établir à l'échelle d'un site si la perte de sol est tolérable, acceptable, ou si elle fait tout simplement partie de l'équilibre naturel du système.

\section{Érosion éolienne}

L'érosion éolienne se rencontre surtout en régions semi-arides (Al Qudah, 2001 ; Badraoui et Stitou 2001 ; Dudeen, 2001 ; Hamdi et Abdelhafez, 2001; Ilaiwi, 2001; Mtimet, 2001 ; López et Arrúe, 2005) où de vastes zones sont devenues, après défrichement et culture, particulièrement sensibles au vent (Skouri, 1993). Le surpâturage est fréquemment cité comme une cause importante du phénomène. Selon Floret et al. (1993), le labour mécanisé introduit dans les années 1920 au Maghreb s'est répandu rapidement dans la steppe ; la surface céréalière s'est notablement accrue dans la steppe tunisienne entre les années 1948 et 1975 et avec elle l'érosion éolienne. Le premier recensement général de l'agriculture en Algérie (2001) révèle que les emblavures de céréales de régions traditionnellement steppiques sont équivalentes à celles de régions traditionnellement céréalières. En Syrie (Ilaiwi, 2001), l'expansion de l'agriculture pluviale dans la steppe a débuté dans les années 1980: elle s'est vite accompagnée d'une érosion éolienne très forte. La fréquence des vents de poussières a été telle que le labour fut interdit dans la steppe en 1995.

L'érosion éolienne semble avoir bénéficié de peu de recherches en Méditerranée. Des travaux entrepris en Aragon (nord-est de l'Espagne), montrent que la texture et le mode de gestion du sol sont les principaux facteurs affectant son érodibilité (López et al., 2001 ; López et Arrúe, 2005). Dans cette même région, Gomes et al. (2003) confirment la faible érodibilité des sols soumis à un travail réduit. Par ailleurs, ils observent l'absence d'érosion éolienne lorsque des croûtes superficielles se forment à la surface du sol, même quand le vent est puissant. Le caractère généralisé des croûtes dans cette région amène les auteurs à recommander plus de recherches sur la stabilité de ces formations afin d'en évaluer l'efficience dans la lutte contre l'érosion éolienne. Cependant, ces croûtes peuvent être une des causes principales du faible taux d'infiltration du sol et de l'augmentation de l'érosion par ruissellement (Boukheir et al., 2001) comme cela a été établi dans les sols du Sahel africain (Casenave et Valentin, 1989).

\section{Dégradations}

\section{de la structure des sols et baisse du taux de matière organique et de l'activité biologique}

Ces types de dégradations sont évoqués dans nombre de publications (Al Qudah, 2001 ; Badraoui et Stitou, 2001 ; Boukheir et al., 2001 ; Giordano et Filippi, 1993). Un faible taux de la matière organique du sol et une structure de faible stabilité sont souvent associés (Caravaca et al., 2002 ; Hernanz et al., 2002).

Selon Six et al. (2004), la dynamique de l'agrégation, la décomposition et la stabilisation de la matière organique du sol, et l'activité biologique du sol sont liées. La formation et la stabilité des agrégats dépendent de nombreux facteurs dont les racines, la faune, les micro-organismes, les liants minéraux et des variables environnementales comme les cycles de geldégel ou d'humectation-dessiccation et le feu. Le travail du sol accélère le rythme de renouvellement des agrégats et diminue la protection de la matière organique du sol (Six et al., 2004). De plus, une gestion qui limite le renouvellement de la matière organique du sol ou l'activité des vers de terre diminue la stabilité des agrégats. Or : i) l'agriculture méditerranéenne utilise amplement le labour et les façons superficielles. La technique du dry farming, basée sur le travail fréquent du sol, est largement utilisée dans les systèmes pluviaux, céréaliers notamment, pour pallier l'insuffisance et la variabilité des pluies ; et ii) le défrichement, la déforestation, le surpâturage et le brûlis des résidus de culture sont amplement rapportés (Skouri, 1993 ; Giordano et Filippi, 1993).

La perte de la matière organique du sol et la réduction de la biodiversité sont fréquemment citées mais peu chiffrées. En Sardaigne (Vacca et al., 2002), les résultats indiquent que le labour de sols de forêt de chêne-liège conduit à des baisses notables du taux de matière organique des horizons de surface, passant de 3,4 à 1,5\%. Dans les hautes terres de Turquie (Celik, 2005), un sol de prairie cultivé perd en moyenne, en 12 ans, la moitié de la teneur en matière organique originelle des 20 premiers centimètres. Parallèle- 
ment, sa densité apparente augmente et la stabilité à l'eau de ses agrégats diminue. Dans les périmètres irrigués du semi-aride marocain, la perte moyenne de la matière organique du sol est voisine de $50 \%$ sur une durée de mise en eau de 30 ans (Naman et al., 2001). La matière organique diminue fortement dans les sols conduits en dry farming, et des taux annuels de minéralisation de l'humus de l'ordre $5 \%$ ont été atteints dans des sols finement pulvérisés (Mhiri et Bousnina, 1998).

Dans les agroécosystèmes semi-arides méditerranéens des croûtes se forment fréquemment à la surface du sol. Leur présence indiquerait à la fois une faible stabilité des agrégats, la diminution de la matière organique du sol et de l'activité biologique favorisés par le travail du sol. En revanche, des recherches récentes montrent que la réduction du travail du sol et l'utilisation d'outils qui ne retournent pas et ne pulvérisent pas le sol, le zéro labour, la couverture du sol par les résidus de cultures et les amendements organiques contribuent à stabiliser les agrégats et à relever le taux de la matière organique et l'activité biologique des sols (Kribaa et al., 2001 ; Mrabet et al., 2001 ; Hernanz et al., 2002 ; Pagliai et al., 2004 ; Moreno et al., 2006). Le maintien de résidus de cultures à la surface du sol est déterminant dans la réduction des croûtes superficielles (Usón et Poch, 2000).

En raison de leur impact sur l'eau notamment (Casenave et Valentin, 1989; Eldridge et al., 2000), les formations de croûtes superficielles méritent une attention toute particulière en milieu méditerranéen.

\section{Dégradations chimiques}

Les dégradations précédemment décrites affectent les propriétés chimiques des sols et leur fertilité. Mais ces aspects demeurent peu documentés en Méditerranée.

Les données sur la consommation d'engrais des pays méditerranéens (United Nations Food and Agriculture Organisation, 2005) suggèrent la coexistence de deux agricultures : l'une minière, généralement pluviale, puisant dans la fertilité des sols et l'épuisant, l'autre surfertilisée, généralement irriguée, amenant avec elle des pollutions. Badraoui et al. (2002) notent que des formules générales de fertilisation ont été utilisées dans les périmètres irrigués du Maroc pendant longtemps sans suivi de l'évolution de la fertilité des sols. Dinç et al. (2001),
Dudeen (2001), Giordano et Filippi (1993) et Vella, 2001 évoquent l'excès de fertilisants et des pollutions par des métaux lourds. Les pollutions par les pesticides sont aussi soulignées (Giordano et Filippi, 1993 ; Dudeen, 2001 ; Hamdi et Abdelhafez, 2001). Des pollutions issues des déchets urbains (Hamdi et Abdelhafez, 2001 ; Zdruli et Lushaj, 2001) et des contaminations par des métaux lourds provenant d'anciennes mines abandonnées (Zdruli et Lushaj, 2001 ; Vacca et al., 2002) sont rapportées.

Les dégradations chimiques, les pollutions en particulier et leurs risques pour la chaîne alimentaire et la santé devraient susciter plus d'attention.

\section{Pertes de sol dues à l'urbanisation, aux infrastructures et aux activités non agricoles}

La pression sur les sols engendrée par l'accroissement de la population est une problématique ancienne en Méditerranée. Les Grecs et les Étrusques l'ont résolue en augmentant leurs surfaces agricoles grâce au terrassement des pentes fortes, au drainage et à l'aménagement des marécages et zones inondables (Griesbach, 1993). De nos jours, la réponse à la pression démographique se traduit notamment par la croissance des espaces urbains et avec eux les infrastructures industrielles, commerciales, de transport, d'artisanat et de loisirs, aux dépens de sols facilement accessibles et généralement de haut potentiel agricole (Darwish, 2001 ; Dinç et al., 2001 ; Hadjiparaskevas, 2001 ; Hamdi et Abdelhafez, 2001 ; Mtimet, 2001 ; Vacca et al., 2002 ; Zdruli et Lushaj, 2001). Au lieu de gagner des surfaces en sols cultivables, il semble qu'on en perde, et que les surfaces perdues concernent les meilleurs sols : ceux-là mêmes qui, à l'origine, avaient pour fonction de nourrir les villes. Ainsi, l'accroissement de la ville de Barcelone a consommé entre 1982 et 1989 près de 15000 hectares de terres agricoles et 7000 hectares de terres forestières (De Torres i Capell et Sagarra i trias, 1993). En Égypte, l'urbanisation devient le problème numéro un; les pertes annuelles de sol y sont estimées à près de 61000 hectares (Hamdi et Abdelhafez, 2001).

À ces pertes de sol par occupation et scellement de l'espace, s'ajoutent des per- tes dues à des activités telles que l'extraction de matériaux, qui détruisent complètement le sol. En Sardaigne (Vacca et al., 2002), l'extraction de sables et de graviers des plaines alluviales a complètement changé le paysage et conduit à la perte de sols à haut potentiel agricole. En Égypte, Hamdi et Abdelhafez (2001) évoquent la fabrication industrielle de briques, qui, au rythme de l'accroissement de l'habitat, consommerait des quantités considérables de terre.

L'accroissement de la population, notamment au sud et à l'est de la Méditerranée est encore loin d'être maîtrisé. Les besoins subséquents en habitat et en infrastructures laissent penser que les pertes en sol vont se poursuivre. Or les ressources en sols y sont déjà rares : dans le meilleur des cas, la surface de terre arable par habitant ne dépasse pas 0,36 hectare selon les chiffres de la FAO (United Nations Food and Agriculture Organisation, 2005).

\section{Conclusion}

Les quelques faits rapportés ci-dessus indiquent que la question de la dégradation des sols est généralisée autour de la Méditerranée mais qu'elle reste encore peu documentée; sa sévérité et ses risques sont encore loin d'être rigoureusement évalués. Si la problématique émerge, elle n'est pas encore perçue au rang des enjeux de développement. Les raisons en sont nombreuses ; en particulier, la question des sols a jusqu'ici été traitée, aussi bien au niveau national qu'à l'échelle internationale, dans des cadres thématiques et sectoriels alors qu'elle nécessite une approche systémique comme l'ont souligné Hamdy et al. (2002). Il en a résulté une multiplication des acteurs, des outils et des méthodes d'études et d'approche des sols, parfois au sein d'un même pays, aboutissant à des résultats difficilement comparables et exploitables sans parler de la duplication des efforts et de l'envoi de messages parfois contradictoires vers les décideurs. A cela on doit ajouter le manque d'une définition claire et largement partagée de la dégradation des sols, de ses indicateurs et des moyens de la quantifier.

Même si elle est pionnière en la matière, l'agriculture n'est plus la seule activité qui dégrade les sols autour de la Méditerranée. Tous les autres secteurs d'activité et 
l'urbanisme, parfois chaotique, contribuent largement à la raréfaction des ressources en sols et à leur dégradation. D'une côte à l'autre de la Méditerranée, l'impératif d'un développement durable incite à une révision profonde des modes actuels d'utilisation et de gestion des sols; il s'agit d'aller au-delà d'une simple attitude de préservation des sols, vers une réelle politique méditerranéenne de gestion durable des sols. En effet :

- une prise en charge de la dégradation des sols nécessite indiscutablement un effort continu de recherche et des financements, dépassant le cadre et les moyens d'un seul État ;

- dans tout l'ensemble méditerranéen on retrouve les mêmes conditions de milieu. L'utilisation de ces milieux par l'homme a conduit partout aux mêmes formes de dégradation. Ainsi, des stratégies de coopération bâties sur la complémentarité augmenteraient l'efficacité de la recherche tout en limitant ses coûts ;

- il n'y a pas actuellement d'institution méditerranéenne internationale dont le mandat soit consacré au sol, où peuvent être élaborées les priorités et les stratégies de recherche et de formation en Méditerranée.

Une politique méditerranéenne en faveur des sols devrait être fondée sur : i) une nouvelle approche du sol, systémique, s'appuyant sur la multifonctionnalité des sols (Blum, 1998 ; Ruellan 2006) ; ii) des stratégies coopératives innovantes, notamment pour ce qui concerne la génération (recherche) et le partage (éducation, formation et popularisation) des connaissances et impliquant l'ensemble des acteurs concernés par le sol ; et iii) un cadre institutionnel méditerranéen approprié, relayé dans chacun des pays par une structure ayant mandat national sur les sols, centralisant et disséminant les connaissances et apportant la caution scientifique aux politiques relatives aux sols.

\section{Références}

Al-Qudah B. Soils of Jordan. In : Zdruli P, Steduto $P$, Lacirignola C, Montanarella $L$, eds. Soil resources of Southern and Eastern Mediterranean countries. Bari : Centre international de hautes études agronomiques méditerranéennes-Instituto Agronomico Mediterraneo di Bari (Ciheam-IAMB), 2001.

Badraoui M, Albani M, Agbani M, et al. New references for the fertilisation of wheat, sugar beet, and sunflower in Doukkala and Gharb irrigated perimeters in Morocco. In : Zdruli P, Steduto P, Kapur S, eds. 7th international meeting on soils with Mediterranean type of climate. Bari : Centre international de hautes études agronomiques méditerranéennesInstituto Agronomico Mediterraneo di Bari (Ciheam-IAMB), 2002.

Badraoui M, Stitou M. Status of soil survey and soil information system in Morocco. In Zdruli P, Steduto P, Lacirignola C, Montanarella $L$, eds. Soil resources of Southern and Eastern Mediterranean countries. Bari : Centre international de hautes études agronomiques méditerranéennes-Instituto Agronomico Mediterraneo di Bari (Ciheam-IAMB), 2001

Badraoui M, Soudi B, Ferhat A. Variation de la qualité des sols: une base pour évaluer la durabilité de la mise en valeur agricole sous irrigation par pivot au Maroc. Etude et Gestion des Sols $1998 ; 5$ : 227-34.

Blum WEH. Les différentes fonctions du sol. In : Lahmar R, Ribaut JP, eds. Sols et sociétés. Regards pluriculturels. Paris : éditions Charles Léopold Mayer, 1998.

Boukheir R, Girard M-C, Khawlie M, Abadallah C. Erosion hydrique des sols dans les milieux méditerranéens : une revue bibliographique. Etude et Gestion des Sols 2001; 8 : 231-45.

Boulaine J. Facteurs de formation des sols méditerranéens. Sols Afr 1961 ; VI : 249-72.

Caravaca F, Masciandaro G, Ceccanti B. Land use in relation to soil chemical and biochemical properties in a semiarid Mediterranean environment. Soil Till Res 2002 ; 68 : 23-30.

Casenave A, Valentin C. Les états de surface de la zone sahélienne. Influence sur l'infiltration. Paris : Orstom éditions, 1989.

Celik I. Land use effects on organic matter and physical properties of soil in a southern Medi-
terranean highland of Turkey. Soil Till Res terranean highland $2005 ; 83: 270-7$.

Costantini EAC, Barbetti R, Righini G. Managing the uncertainty in soil mapping and land evaluation in areas of high pedodiversity. Methods and strategies applied in the province of Siena (Central Italy). In : Zdruli P, Steduto P, Kapur S, eds. 7th international meeting on soils with Mediterranean type of climate. Bari : Centre international de hautes études agronomiques méditerranéennesétudes agronomiques méditerranéennes(Ciheam-IAMB), 2002.

Daoud Y, Halitim A. Irrigation et salinisation au Sahara algérien. Sécheresse 1994 ; 5 : 151-61.

Darwish T, Khawlie M, Jomaa I, Awad M, Abou-Daher M, Zdruli P. A survey to upgrade information for soil mapping and management in Lebanon. In : Zdruli $P$, Steduto $P$, ment in Lebanon. In : Zdruli P, Steduto P,
Kapur S, eds. 7th international meeting on soils with Mediterranean type of climate. Bari : Centre international de hautes études agronomiques méditerranéennes-Instituto Agronomico Mediterraneo di Bari (Ciheam-IAMB), 2002.

Darwish TM. Status of soil survey in Lebanon. The need for a georeferenced soil database. In : Zdruli $\mathrm{P}$, Steduto $\mathrm{P}$, Lacirignola $\mathrm{C}$ Montanarella $L_{\text {, eds. Soil resources of }}$ Montanarella $\mathrm{L}$, eds. Soil resources of
Southern and Eastern Mediterranean countries. Bari : Centre international de hautes études agronomiques méditerranéennesInstituto agronomico Mediterraneo di Bari (Ciheam-IAMB), 2001
De Torres i Capell M, Sagarra i trias F. The urban impact on soils in the Mediterranean area. In : État de l'agriculture en Méditerranée. Les sols dans la région méditerranéenne : utilisation, gestion et perspectives d'évolution. Zaragoza: Centre international de hautes études agronomiques méditerranéennesInstituto Agronómico Mediterráneo de Zaragoza (Ciheam-IAMZ), 1993.

Dinç U, Kapur S, Akça E, et al. History and status of soil survey programmes in Turkey and suggestions on land management. In : Zdruli $P$, Steduto $P$, Lacirignola C, Montanarella $L$, eds. Soil resources of Southern and rella $L$, eds. Soil resources of Southern and
Eastern Mediterranean countries. Bari : Centre international de hautes études agronomiques méditerranéennes-Instituto Agronomico Mediterraneo di Bari (Ciheam-IAMB), 2001.

Dosso M. Géochimie des sols salés et des eaux d'irrigation. Aménagement de la Basse Vallée de l'Euphrate en Syrie. Thèse docteuringénieur, université Paul Sabatier, Toulouse, 1980.

Dudeen B. The soils of Palestine (The West Bank and Gaza Strip) current status and future perspectives. In : Zdruli $P$, Steduto $P$, Lacirignola C, Montanarella L, eds. Soil resources of Southern and Eastern Mediterranean countries. Bari: Centre international de hautes études agronomiques méditerranéennesétudes agronomiques méditerranéennesInstituto Agronomico
(Ciheam-IAMB), 2001.

Eldridge DJ, Zaady E, Shachak M. Infiltration through three contrasting biological soil crusts in patterned landscape in the Negev, Israel. CATENA $2000 ; 40: 323-36$.

Eswaran H, Reich P. A preliminary assessment of the human impact on land systems of the world. Transactions of the 17th WCSS Bangkok, CDrom paper 2128, 2002.

Eswaran H, Lal R, Reich PF. Land degradation: an overview. In: Bridges EM, et al., eds. Responses to land degradation. New Delhi : Oxford Press, 2001.

Floret C, Le Floch E, Pontanier R. Agriculture and desertification in arid zones of North Africa. In : État de I'agriculture en Méditerranée. Les sols dans la région méditerranéenne: utilisation, gestion et perspectives d'évolution. Zaragoza : Centre international de hautes études agronomiques méditerranéennesInstituto Agronómico Mediterráneo de Zaragoza (Ciheam-IAMZ), 1993.

Giordano A, Filippi N. Advantages and disadvantages of land use changes for the preservation of soil resources. Review of soil conservation practices and the need for related research. In: État de l'agriculture en Méditerranée. Les sols dans la région méditerranéenne: utilisation, gestion et perspectives d'évolution. Zaragoza : Centre international de hautes études agronomiques méditerranéennes-Instituto Agronómico Mediterráneo de Zaragoza (Ciheam-IAMZ), 1993.

Gomes L, Arrúe JL, López MV, et al. Wind erosion in semiarid agricultural area of Spain : the WELSONS project. CATENA $2003 ; 52$ : 235-56.

Griesbach JC. The present state of soil resources in the Mediterranean countries. In : Etat de l'agriculture en Méditerranée. Les sols dans la région méditerranéenne : utilisation, gestion et perspectives d'évolution. Zaragoza: Centre international de hautes études agronomiques méditerranéennesInstituto Agronómico Mediterráneo de Zaragoza (Ciheam-IAMZ), 1993. 
Hadjiparaskevas C. Soil survey in Cyprus. In : Zdruli P, Steduto P, Lacirignola C, Montanarella $\mathrm{L}$, eds. Soil resources of Southern and Eastern Mediterranean countries. Bari : Centre international de hautes études agronomiques méditerranéennes-Instituto Agronomico Mediterraneo di Bari (Ciheam-IAMB), 2001.

Hamdi $\mathrm{H}$, Abdelhafez S. Agriculture and soil survey in Egypt. In : Zdruli P, Steduto P, Lacirignola C. Montanarella L, eds. Soil resources of Southern and Eastern Mediterranean coun tries. Bari: Centre international de hautes études agronomiques méditerranéennesInstituto Agronomico Mediterraneo di Bar (Ciheam-IAMB), 2001

Hamdy A, Lacirignola C, Trisorio-Liuzzi G. The integration of soil and water resources management towards a sustainable agricultura development in the Mediterranean. In Zdruli P, Steduto P, Kapur S, eds. 7th interna tional meeting on soils with Mediterranean type of climate. Bari : Centre international de hautes études agronomiques méditerranéennes-Instituto Agronomico Mediterraneo di Bari (Ciheam-IAMB), 2002.

Hernanz JL, López R, Navarette L, SánchezGirón V. Long-term effects of tillage systems and rotations on soil structural stability and organic carbon stratification in semiarid central Spain. Soil Till Res 2002 ; 66 : 129-41.

Ilaiwi M. Soils of the Syrian Arab Republic. In Zdruli P, Steduto P, Lacirignola C, Montanarella $L$, eds. Soil resources of Southern and Eastern Mediterranean countries. Bari : Centre international de hautes études agrointernational de hautes études agronomico Mediterraneo di Bari (Ciheam-IAMB), 2001

Kribaa M, Hallaire V, Curmi P, Lahmar R. Effect of various cultivation methods on the structure and hydraulic properties of a soil in a semi-arid climate. Soil Till Res 2001; 60 43-53.

Lavee $\mathrm{H}$ Calvo-Cases $\mathrm{A}$ Sarah $\mathrm{P}$ BoixFayos C, Ben-Shmuel M, Arnau-Rosalen E. Lessons and experiences gained from 20 years of measuring soil erosion and related data in the Mediterranean : Future challenges and the way ahead. In : Van Asselen S, Boix-Fayos C Imeson $A$, eds. Briefing papers of the second SCAPE workshop in Cinque Terre (Italy), 2004. www.scape.org.

Lipper L, Osgood D. Two essays on socio economic aspects of soil degradation. FAO Economic and social development paper, 149. Rome: United Nations Food and Agriculture Organisation (FAO), 2001.

López MV, Arrúe JL. Soil tillage and wind erosion in fallow lands of Central Aragaon, Spain : An overview. In : Faz A, Ortiz R, Mermut AR, eds. Sustainable Use and Management of Soils : Arid and Semiarid Regions. Advances in GeoEcology, 36. Reiskirchen (Germany): Catena Verlag Gmbh, 2005.

López MV, Gracia R, Arrúe JL. An evaluation of wind erosion hazards in fallow lands of semiarid Aragon (NE Spain). J Soil Water Cons $2001 ; 56: 212-9$.
Malik SJ. Rural poverty and land degradation A reality check for the CGIAR. CGIAR Technical Advisory Committee and FAO. Rev.1. Rome United Nations Food and Agriculture organisation (FAO), 1998. www.fao.org/Waidocs/ TAC/x5784E/x5784e0d.htm

MhiriA, Bousnina H.Diagnosticagri-environnemental de l'état des terres cultivées dans les divers systèmes de production en Tunisie. In Mhiri A, ed. Mobilisation, exploitation et conservation des ressources naturelles. Tunis : Institut national agronomique de Tunisie (Inat), 1998.

Mhiri A, Tarhouni J, Hachicha M, Lebdi F. Approche systémique des risques de salinisation par andoréisation anthropique. Etude et Gestion des Sols 1998 ; 5 : 257-68.

Moreno F, Murillo JM, Pelegrín F, Girón IF. Long-term impact of conservation tillage on stratification ratio of soil organic carbon and loss of total and active $\mathrm{CaCO}_{3}$. Soil Till Res $2006 ; 85: 86-93$

Mrabet R, Saber N, El-Brahli A, Lahlou S, Bessam F. Total particulate organic matter, and structural stability of a calcixeroll soil under different wheat rotations and tillage systems in a semi-arid area of Morocco. Soil Till Res $2001 ; 57: 225-35$

Mtimet A. Soils of Tunisia. In : Zdruli P, Steduto $\mathrm{P}$, Lacirignola $C$, Montanarella $L$, eds. Soil resources of Southern and Eastern Mediterranean countries. Bari : Centre international de hautes études agronomiques méditerranéennes-Instituto Agronomico Mediterraneo di Bari (Ciheam-IAMB), 2001.

Nachtergaele FO, Oldeman LR. World soil and terrain database (SOTER) : past, present and future. Transactions of the 17th WCSS Bangkok, CDrom paper 653, 2002.

Naman F, Soudi B, Chiang C. Impact de l'intensification agricole sur le statut de la matière organique des sols en zones irrigues semiarides au Maroc. Etude et Gestion des Sols arides au Maroc.

Oldeman LR, Hakkeling RTA, Sombroek WG. World map of the status of human-induced soil degradation. An explanatory Note. Global Assessment of Soil Degradation. Wageningen : International Soil Reference and Information Centre (ISRIC) : United Nations Environment Program (UNEP), 1991.

Pagliai M, Vignozzi N, Pellegrini S. Soil structure and the effect of management practices. Soil Till Res $2004 ; 79$ : 131-41.

Pardini G, Gispert M, Dunjo G. Runoff erosion and nutrient depletion in five Mediterranean soils of NE Spain under different land use. Sci Total Environ 2003 ; 39 : 213-24.

Ramdane M. Agro-pedological studies in Algeria. In : Zdruli P, Steduto P, Lacirignola C Montanarella $L$, eds. Soil resources of Southern and Eastern Mediterranean countries. Bari: Centre international de hautes études agronomiques méditerranéennes Instituto Agronomico Mediterraneo di Bari (Ciheam-IAMB), 2001

Roose E, De Noni G. Apport de la recherche à la lutte antiérosive. Bilan mitigé et nouvelle approche. Etude et Gestion des sols 1998 ; 5 : 181-94.
Ruellan A. Sols et développement durable. L'Encyclopédie du développement durable. Paris : éditions des Récollets, 2006.

Scherr SJ. Soil degradation. A threat to developing-country food security by 2020? Food, agriculture, and the environment discussion paper 27. Washington (DC) : International Food Policy Research Institute (IFPRI) 1999.

Skouri M. La désertification dans le bassin méditerranéen : état actuel et tendance. In : Etat de l'Agriculture en Méditerranée. Les sols dans la région méditerranéenne: utilisation, gestion et perspectives d'évolution. Zaragoza : Centre international de hautes études agronomiques méditerranéennes-Instituto Agronómico Mediterráneo de Zaragoza (CiheamIAMZ), 1993.

Six J, Bossuyt H, Degryze S, Denef K. A history of research on the link between (micro) aggregates, soil biota, and soil organic matter dynamics. Soil Till Res 2004 ; 79 : 7-31.

United Nations Food and Agriculture Organisation (FAO). La situation mondiale de l'alimentation et de l'agriculture. Le commerce agricole et la pauvreté : le commerce peut-il être au service des pauvres?. Rome: FAO 2005.

Usón A, Poch RM. Effects of tillage and management practices on soil crust morphology under a Mediterranean environment. Soil Till Res $2000 ; 54: 191-6$.

Vacca A, Loddo S, Serra G, Aru A. Soil degradation in Sardinia (Italy): main factors and processes. In : Zdruli $\mathrm{P}$, Steduto $\mathrm{P}$, Kapur $\mathrm{S}$ eds. 7th international meeting on soils with Mediterranean type of climate. Bari : Centre international de hautes études agronomiques méditerranéennes-Instituto Agronomico Mediterraneo di Bari (Ciheam-IAMB), 2002.

Van Lynden GWJ. La ressource sol en Europe. Strasbourg: les éditions du Conseil de I'Europe, 1995.

Vella S. Soil information in the Maltese Islands. In : Zdruli P, Steduto P, Lacirignola C, Montanarella L, eds. Soil resources of Southern and Eastern Mediterranean countries. Bari : Centre international de hautes études agronomiques méditerranéennesInstituto Agronomico Mediterraneo di Bari (Ciheam-IAMB), 2001.

Yaalon $\mathrm{DH}$. Soils in the Mediterranean region : what makes them different? CATENA 1997 28 : 157-69.

Zdruli P, Lushaj S, Pezzuto A, et al. Preparing a georeferenced soil database for Albania at scale $2: 250,000$ using the European soil bureau manual of procedures 1.1. In : Zdruli $P$, Steduto P, Kapur S, eds. 7th international meeting on soils with Mediterranean type of climate. Bari: Centre international de hautes études agronomiques méditerranéennesInstituto Agronomico Mediterraneo di Bari (Ciheam-IAMB), 2002

Zdruli $P$, Lushaj $S$. The status of soil survey in Albania and some of its major environmental findings. In : Zdruli P, Steduto P, Lacirignola C, Montanarella $L$, eds. Soil resources of Southern and Eastern Mediterranean countries. Bari: Centre international de hautes études agronomiques méditerranéennesInstituto Agronomico Mediterraneo di Bari (Ciheam-IAMB), 2001 